\title{
Modeling of a Double-Pass Backward Er-Doped Superfluorescent Fiber Source for Fiber-Optic Gyroscope Applications
}

\author{
Lon A. Wang and C. D. Su
}

\begin{abstract}
The characteristics including mean wavelength stability, linewidth, and output power of a double-pass backward (DPB) Er-doped superfluorescent fiber source (SFS) are theoretically analyzed in details. The effects of the variations in fiber length, pump wavelength, fiber mirror reflectance, optical feedback, and erbium concentration on the characteristics are investigated. The analysis results show that an SFS in DPB configuration may serve as light source for the navigation-grade fiber-optic gyroscope applications.
\end{abstract}

Index Terms-Broad-band light sources, Er-doped fiber (EDF), superfluorescent fiber sources (SFS's).

\section{INTRODUCTION}

$\mathbf{T}$ REMENDOUS progress has been made in the past 20 years on fiber-optic gyroscopes (FOG's). The introduction of new light sources and continued efforts in improving their performance for various FOG applications plays an important role for such progress. It is known the accuracy of rotation detection of an FOG is determined by the stability of scale factor, which then depends on the mean wavelength stability of its light source. For an inertial-navigation grade FOG, a highly stable mean wavelength operation, e.g., $\sim 1$ ppm, is required [1]. In addition, broad bandwidth and high output power are the other two desirable characteristics of the light sources required for such applications. High output power can help in two ways. First, a superfluorescent fiber source (SFS) is thus excess-limited [2], and the signal-to-noise ratio (SNR) is only determined by the source and detector bandwidths, which then manifests itself the importance of broad bandwidth of a light source. Second, it helps relax the sensitivity requirement in a measurement system by allowing the increase of fiber length in a loop.

Although semiconductor light sources such as light-emitting diodes and superluminescent diodes would provide broad bandwidth and sufficient power, they are generally not suitable for the navigation grade FOG applications because of poor spectrum stability, typically of $400 \mathrm{ppm} /{ }^{\circ} \mathrm{C}$ [1]. In contrast, temperature stability and output power of diode pumped rare-

Manuscript received July 14, 1999. This work was supported by the National Science Council, Taiwan, R.O.C., under Contracts CS 84-0210-D002-008 and NSC 88-2215-E-002-004.

L. A. Wang is with the Institute of Electro-Optical Engineering, National Taiwan University, Taipei, Taiwan, R.O.C. He is also with the Department of Electrical Engineering, National Taiwan University, Taipei, Taiwan, R.O.C.

C. D. Su is with the Institute of Electro-Optical Engineering, National Taiwan University, Taipei, Taiwan, R.O.C.

Publisher Item Identifier S 0733-8724(99)09039-8. earth-doped SFS's can be a few orders of magnitude better than the semiconductor counterparts. However, characteristics of an SFS strongly depend on which arrangement or configuration is used, and has lately attracted much attention in order to find an optimal performance for each configuration [3]-[7].

In general, by configurations the SFS's can be classified into four basic kinds and their derivatives. The four basic kinds are of single-pass forward and backward, double-pass forward and backward SFS's. As for single-pass forward SFS's, although there are some reports [5], [8] they are of less interest because of insufficient output power. In contrast, the singlepass backward one, denoted as SPB, has been most commonly adopted owing to its ease in design and less susceptibility to lasing [3]-[6]. The stable mean wavelength operation for SPB SFS's when pumped near $980 \mathrm{~nm}$ was first studied by Wysocki et al. [5], [9], and then experimentally analyzed in details by Hall et al. [4]. Compared to single-pass SFS's, fewer results have been reported on double-pass SFS's [5], [10]-[11] particularly for the double-pass backward one denoted as DPB, since they are more susceptible to the resonant lasing in the presence of external feedback. The previous reports on DPB SFS's can be summarized as the following.

The DPB SFS was demonstrated by Dulling III et al. [12], [13] who utilized a frequency-independent rigorous analysis for the calculation of output power and threshold feedback. However, such analysis cannot be applied to the study of mean wavelength and linewidth variations that involves frequencydependent power evolution. Though output [12], [13] and 3-dB bandwidth [12] at different pump power were measured, other important parameters such as mean wavelength stability, isolation required for feedback reduction to maintain stable broadband operation, etc., are not included in these work. Note the term "feedback" used in [4] to describe the signal from the unpumped fiber end of an SPB SFS is functionally the same as mirror reflectance in a DPB SFS. The effect of mirror reflectance on the mean wavelength was briefly mentioned in [4] since the focus there was on an SPB SFS. Recently, DPB SFS's have been experimentally shown to have a large output power, broadband, and stable operation [7], [14]-[15]. Other SFS works on spectrum broadening in the DPB configuration have also been reported [16], [17]. However, to the best of our knowledge, very few simulation results up to date have been reported on Er-doped DPB SFS's pumped near $980 \mathrm{~nm}$.

In this paper, the power evolution equation is employed to simulate the effects of pump power, Er-doped fiber (EDF) 
length, pump wavelength, mirror reflectance, optical feedback, and erbium concentration on the characteristics of a DPB SFS including mean wavelength stability, output power and linewidth. How to reduce the overall thermal effect on the mean wavelength stability is also studied. The rest of the paper is arranged as follows. In Section II, we briefly review the dependence of mean wavelength stability. In Section III, the modeling and the simulation results on the three main characteristics are described, then followed by further discussions on how the characteristics would be affected by related parameters. Finally, we conclude our study in Section IV.

\section{DePENDENCE of MeAn WaVElength Stability}

Since the mean wavelength stability determines the accuracy of rotation detection, and is therefore regarded as the most important parameter of an SFS for a navigation-grade FOG. The mean wavelength of an SFS is temperature dependent, and has three contributing sources: intrinsic thermal effect, pumpwavelength related and pump-power related factors. They can be expressed as the summation of three terms as follows:

$$
\begin{aligned}
\frac{d \bar{\lambda}_{\text {source }}}{d T}= & \left(\frac{\partial \bar{\lambda}_{\text {source }}}{\partial T}\right)_{\text {int. }}+\left(\frac{\partial \bar{\lambda}_{\text {source }}}{\partial \bar{\lambda}_{\text {pump }}}\right)\left(\frac{\partial \bar{\lambda}_{\text {pump }}}{\partial T}\right) \\
& +\left(\frac{\partial \bar{\lambda}_{\text {source }}}{\partial P_{\text {pump }}}\right)\left(\frac{\partial T_{\text {pump }}}{\partial T}\right) .
\end{aligned}
$$

It is always desirable to have a temperature independent $\left(d \bar{\lambda}_{\text {source }} / d T=0\right)$ or insensitive $\left(d \bar{\lambda}_{\text {source }} / d T \approx 0\right)$ mean wavelength operation, which then requires an optimization among the parameters associated with these three effects. The first term is due to the intrinsic thermal effect, and has a typical value of less than $10 \mathrm{ppm} /{ }^{\circ} \mathrm{C}$. The $\partial \bar{\lambda}_{\text {pump }} / \partial T$ and $\partial P_{\text {pump }} / \partial T$ in the second and the third terms depend on the pump laser diode used, and can only be controlled through the optimization of laser diode's characteristics. Conversely, $\partial \bar{\lambda}_{\text {source }} / \partial \lambda_{\text {pump }}$ and $\partial \bar{\lambda}_{\text {source }} / \partial P_{\text {pump }}$ can be controlled in many different ways. For example, it has been reported for a single-pass SFS that $\partial \bar{\lambda}_{\text {source }} / \partial \lambda_{\text {pump }}$ can be minimized by operating the pump wavelength closer to its peak absorption wavelength (e.g. near $976 \mathrm{~nm}$ ) [3], [5], [15]. Since $\partial \bar{\lambda}_{\text {source }} / \partial P_{\text {pump }}$ depends on the configuration employed, it has been demonstrated experimentally that pump-power independent mean wavelength operation would be obtained in SPB [4] and DPB [7] configurations. We will show that an optimization among the parameters of EDF length, pump power and mirror reflectance, etc. can lead a DPB SFS to a stable mean wavelength operation with high output power and broad bandwidth.

\section{Modeling AND Simulation Results}

\section{A. Modeling of an Er-Doped DPB SFS}

The spectral power evolution of an Er-doped SFS can be simulated by the interaction of population densities at various energy levels and optical signals including amplified spontaneous emission (ASE) and pump. The characteristics of a DPB SFS are determined by many parameters, which
TABLE I

Related Material Parameters

\begin{tabular}{l|l}
\hline $980 \mathrm{~nm}$ pump-state lifetime $\left(\tau_{\mathrm{p}}\right)$ & $7 \mu \mathrm{s}$ \\
\hline $980 \mathrm{~nm}$ upper-state lifetime $\left(\tau_{\mathrm{u}}\right)$ & $11 \mathrm{~ms}$ \\
\hline fraction of unsaturable ion concentration $\left(\mathrm{N}_{\mathrm{ab}}\right)$ & $3 \%$ \\
\hline Pump-state ESA cross-section & $5 * 10^{-21} \mathrm{~cm}^{2}$ \\
\hline Signal background loss $\left(\alpha_{\mathrm{s}}\right)$ & $0.085 \mathrm{~m}^{-1}$ \\
\hline Pump background loss $\left(\alpha_{\mathrm{p}}\right)$ & $0.09 \mathrm{~m}^{-1}$ \\
\hline
\end{tabular}

can be divided into three categories: material parameters $\left(\mathrm{Er}^{3+}\right.$ concentration, codopant concentration, absorption and emission spectral cross sections, etc.), waveguide parameters (numerical aperture, cut-off wavelength, overlapping of optical field and fiber core areas, etc.), and working parameters (pump wavelength, pump power, EDF length, mirror reflectance, etc.). In this paper, we focus on the study of the characteristics resulted from the variation of working parameters in order to obtain an optimal performance.

In simulation, we assume that the EDF has a numerical aperture of 0.16, and is single-moded when pumped near 980 $\mathrm{nm}$. The radial dependence of the optical signals is accounted for by the calculation of effective signal and pump areas. The spectral cross sections of the ASE described in [3] are utilized throughout our simulation. The results thus obtained agree well with the trend of experiment results [14], [15]. The EDF is assumed to have 140 mole ppm $\mathrm{Er}_{2} \mathrm{O}_{3}$ concentration with 1 mole percent $\mathrm{Al}_{2} \mathrm{O}_{3}$. Other material parameters used are listed in Table I.

The ASE spectrum of an Er-doped SFS is divided into ten regions ranging from 1525 to $1566 \mathrm{~nm}$. In each spectral region there are one forward $P_{s}^{+}\left(z, \nu_{s, i}\right)$ and one backward $P_{s}^{-}\left(z, \nu_{s, i}\right)$ signal waves, and one uni-directional pump wave $P_{P}\left(z, \nu_{p}\right)$. The power evolution for these waves can be expressed as follows, which is similar to the advanced equations described by Wysocki et al. [3], [19] except for the inclusion of background losses.

For ASE signal

$$
\begin{aligned}
\frac{d P_{s}^{ \pm}\left(z, \nu_{s, i}\right)}{d z}= \pm & {\left[\gamma_{s}\left(z, \nu_{s, i}\right) P_{s}^{ \pm}\left(z, \nu_{s, i}\right)\right.} \\
& \left.+\gamma_{e s}\left(z, \nu_{s, i}\right) 2 h \nu_{s, i}\left(\frac{\Delta \nu_{h}}{n}\right)\right]
\end{aligned}
$$

for $980 \mathrm{~nm}$ pumping

$$
\frac{d P_{p}\left(z, \nu_{p}\right)}{d z}=-\gamma_{p}\left(z, \nu_{p}\right) P_{p}\left(z, \nu_{p}\right)
$$

where

$$
\begin{aligned}
\gamma_{s}\left(z, \nu_{s, i}\right)= & \frac{A_{0}}{A_{s}}\left[\sigma_{e}\left(\nu_{s, i}\right) N_{u}(z)-\sigma_{a}\left(\nu_{s, i}\right) N_{l}(z)\right. \\
& \left.\quad-\sigma_{a}\left(\nu_{s, i}\right) N_{a b}\right]-\alpha_{s} \\
\gamma_{e s}\left(z, \nu_{s, i}\right)= & \frac{A_{0}}{A_{s}}\left[\sigma_{e}\left(\nu_{s, i}\right) N_{u}(z)\right] \\
\gamma_{P}\left(z, \nu_{P}\right)= & \frac{A_{0}}{A_{P}}\left[\sigma_{p a}\left(\nu_{P}\right) N_{l}(z)-\sigma_{p e}\left(\nu_{P}\right) N_{P}(z)\right. \\
& \left.\quad+\sigma_{e s a}\left(\nu_{P}\right) N_{P}(z)+\sigma_{p a}\left(\nu_{P}\right) N_{a b}(z)\right]+\alpha_{p}
\end{aligned}
$$




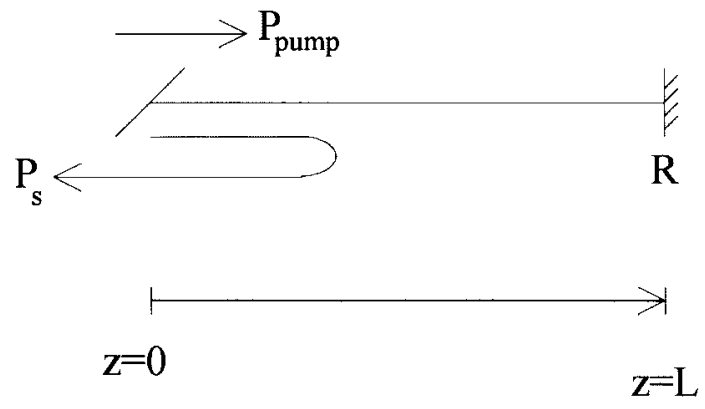

Fig. 1. Schematic diagram of an SFS in DPB configuration.

where

\begin{tabular}{|c|c|}
\hline$A_{0}$ & fiber core area; \\
\hline$A_{s}, A_{P}$ & effective signal mode and pump mode areas \\
\hline$n$ & number of signal regions divided; \\
\hline$\nu_{s, i}$ & signal frequency of $i$ th spectral region; \\
\hline$\nu_{p}$ & pump frequency; \\
\hline$N_{a b}$ & $\begin{array}{l}\text { density of paired erbium ions acting as ab- } \\
\text { sorbers; }\end{array}$ \\
\hline $\begin{array}{l}N_{l}, N_{u} \\
N_{P}\end{array}$ & $\begin{array}{l}\text { population density of lower and upper states; } \\
\text { population density of pump state; }\end{array}$ \\
\hline$\sigma_{p e}, \sigma_{p a}$ & pump emission and absorption cross section; \\
\hline$\sigma_{e s a}$ & pump excited state absorption cross section; \\
\hline$\alpha_{s}, \alpha_{p}$ & signal and pump background loss; \\
\hline$\Delta \nu_{h}$ & ASE signal bandwidth. \\
\hline
\end{tabular}

Loss mechanisms including background losses, pump excited state absorption (ESA), and unsaturable absorbers such as erbium paired ions (assuming as a constant ratio throughout the fiber) are considered to account for the reduction of output power and pump efficiency.

Fig. 1 schematically shows a DPB SFS. Since the feedback from any succeeding port after the output fiber end will affect the characteristics of the SFS, in general an angled fiber end or an isolator should be used to reduce such feedback. The effect of feedback on SFS's characteristics will be discussed in Section III. For an EDF with length $L$, the boundary conditions are $P_{s}^{+}=0$ at $z=0$ and $P_{s}^{-}=R * P_{s}^{+}$at $z=L$. $R$ is the fiber mirror reflectance at the fiber end $z=L$, and is assumed to be $90 \%$ in this paper unless where noted. The characteristics such as mean wavelength, linewidth, and output power are calculated based on the spectral power evolution. The mean wavelength $\bar{\lambda}$ is defined as

$$
\bar{\lambda}=\frac{\sum_{i=1}^{n} P\left(\lambda_{i}\right) \cdot \lambda_{i}}{\sum_{i=1}^{n} P\left(\lambda_{i}\right)}
$$

and, the source bandwidth $\Delta \nu$ is defined as

$$
\delta \nu=\frac{\left[\sum_{i=1}^{n} P\left(\nu_{i}\right) \Delta \nu_{i}\right]^{2}}{\sum_{i=1}^{n} P^{2}\left(\nu_{i}\right) \Delta \nu_{i}} .
$$

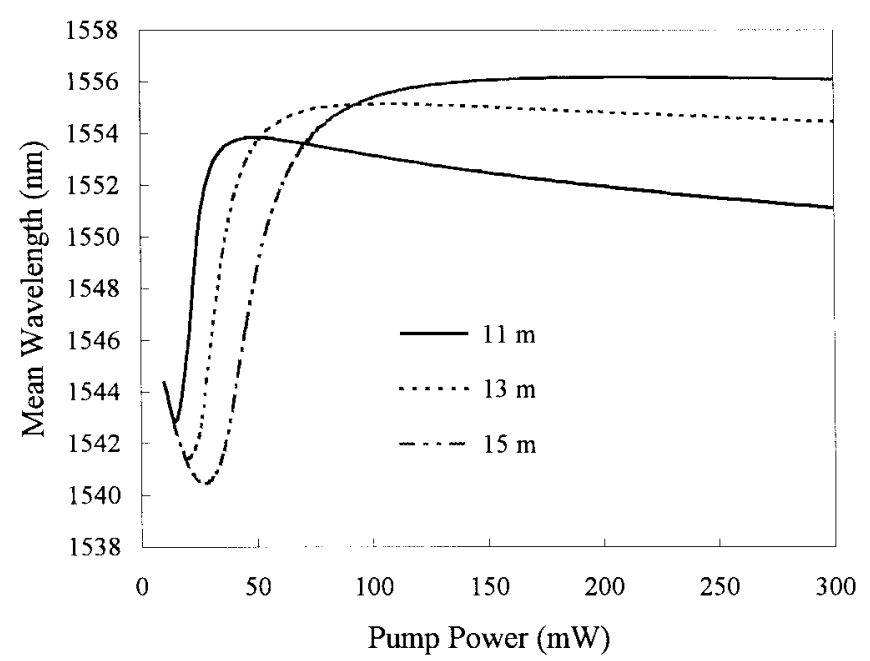

(a)

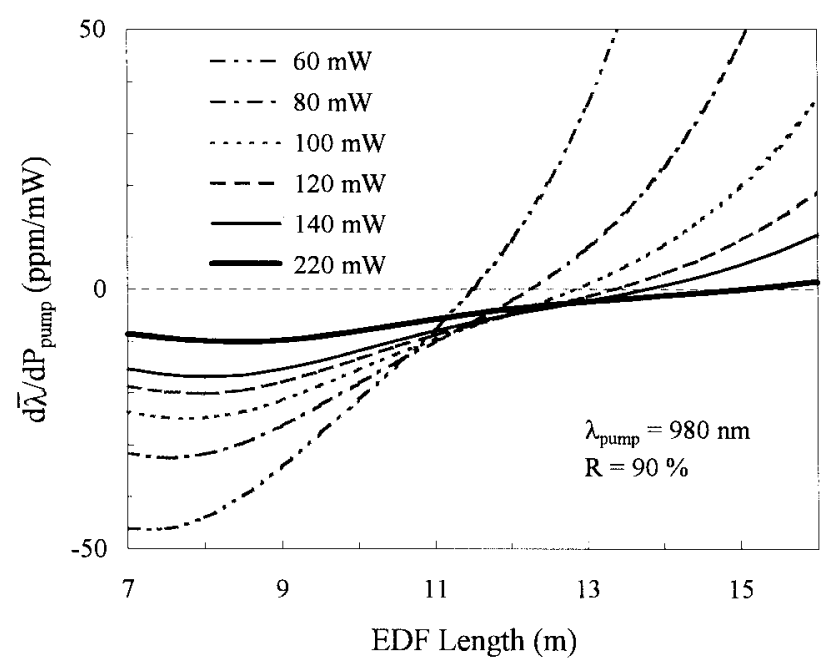

(b)

Fig. 2. (a) Pump-power dependent mean wavelength variation at various EDF lengths and (b) pump-power dependent mean wavelength stability versus EDF length at various pump power.

\section{B. Pump-Power Dependent Mean Wavelength Variation}

The mean wavelength variation as a function of pump power for the SFS's at various EDF lengths is shown in Fig. 2(a). It clearly indicates that a DPB SFS can have pump power independent mean wavelength operation, i.e. $\partial \bar{\lambda}_{\text {source }} / \partial P_{\text {pump }}=0$, in two pump regions: one in low and the other in high pumps. As the EDF length increases, both regions shift toward the high-pump side, indicating that more pump power is needed for such operation. For a given EDF length, the mean wavelengths in high-pump regions are much more stable than those in low-pump ones as evidenced by comparing both slope variations. Thus plateaus are formed in the high-pump regions where the slopes are zero or nearly zero. The plateau location is a function of EDF length and pump power, and can therefore be tuned. Besides, the range of each plateau increases with EDF length, also implying an increase of pump power tolerance. Fig. 2(b) shows the corresponding variation of mean wavelength stability. The optimal EDF length, defined as the length at which $\partial \bar{\lambda}_{\text {source }} / \partial P_{\text {pump }} \approx 0$ 


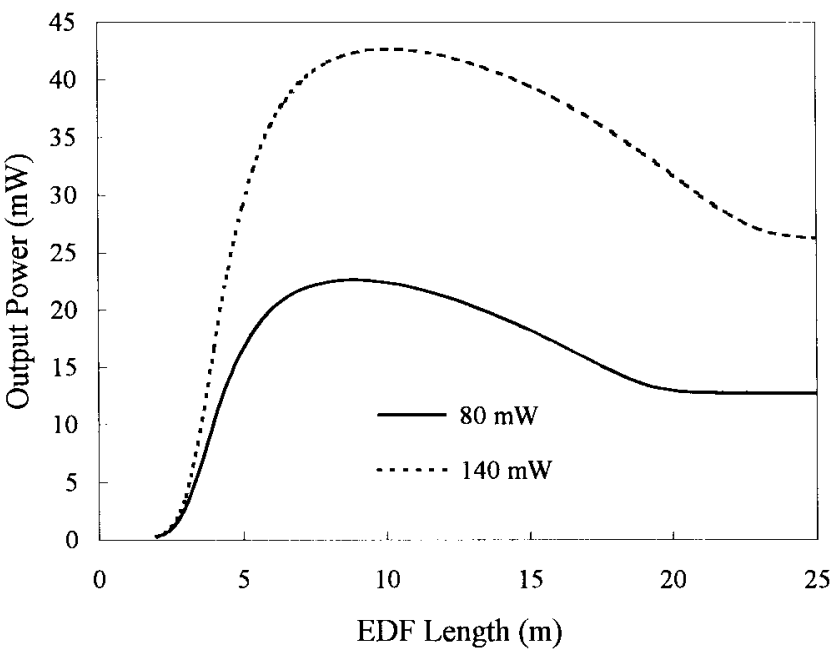

Fig. 3. Output power versus EDF lengths.

occurs, increases slowly with pump power. The slope around each optimal EDF length denotes the tolerance of EDF length for $\partial \bar{\lambda}_{\text {source }} / \partial P_{\text {pump }} \approx 0$. The smaller the slope is, the larger the tolerance becomes. It is seen that the tolerance of optimal EDF length increases markedly with pump power. From Fig. 2(a) and (b), it is found that the operation in higher pump power is desirable, which results in larger tolerances in both the optimal EDF length and pump power range for $\partial \bar{\lambda}_{\text {source }} / \partial P_{\text {pump }} \approx 0$ operation.

\section{Output Power, Pump Efficiency and Linewidth}

The output power as a function of EDF length is shown in Fig. 3 for 80 and $140 \mathrm{~mW}$ pumping. The output power increases rapidly with EDF length, and reaches the maximum value for each pump power. The required EDF lengths to have the maximum output power are shown similar. For output power consideration, the important thing is to have an EDF length exceed the maximum value for a given pump power. Shorter length is an apparent disadvantage because of less output power available. Conversely, longer length causes output power to gradually decrease to a saturated value. The decrease of output power is attributed to the diminishing effectiveness of mirror reflectance.

Fig. 4 shows the relationship of output and pump power. As mentioned previously, there is an optimal EDF length for a given high pump power. Two optimal EDF lengths of 12 and $14 \mathrm{~m}$ are chosen, which correspond to 80 and $140 \mathrm{~mW}$ pumps. The slope efficiencies are $\sim 37$ and $\sim 36 \%$, but become 50 and $55 \%$ when the background losses of signal and pump are excluded. The pump efficiencies are high partly because the pump is injected closer to the signal output end where it is absorbed more rapidly, and partly because the ASE signals travel twice in the EDF. It is noteworthy that when the SFS is operated at its optimal EDF length, the output power is large enough that only one single SFS may serve three-axis, navigation-grade FOG applications.

The variations of threshold pump power and pump efficiency with EDF length are shown in Fig. 5. The threshold pump power is defined as the intercept of the linear line and

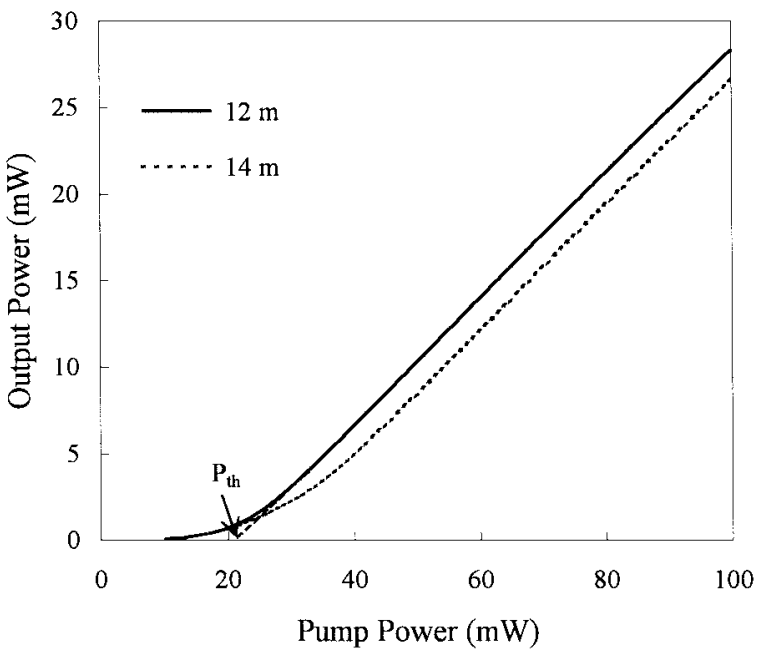

Fig. 4. Output power versus pump power.

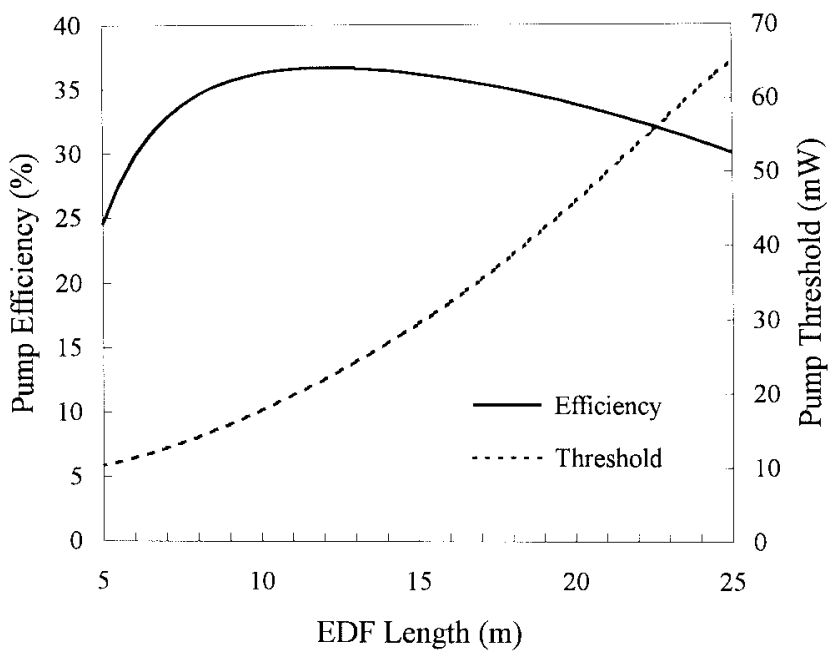

Fig. 5. Pump efficiency and pump threshold versus EDF length.

horizontal axis as shown in Fig. 4. Since the pump power is absorbed rapidly at its input end, there exists some EDF length where the pump power decays to zero and the backward ASE signal ceases to increase. The output power and pump efficiency therefore first increase then decrease to reach the saturated values that are in fact the same as the SPB SFS. The threshold pump power however increases monotonically with the EDF length. Also note that when the optimal EDF lengths are chosen, the pump efficiencies are almost at their maxima.

A typical linewidth and mean wavelength variation with EDF length at $80 \mathrm{~mW}$ pump are shown in Fig. 6. As the EDF length increases, the linewidth repeatedly increases and decreases before reaching a saturation level. Such behavior can be explained from the evolution of two major bands, one is $\sim 1532 \mathrm{~nm}$ and the other $\sim 1558 \mathrm{~nm}$, in ASE spectra, and is also manifested itself in the mean wavelength variation. The relative intensity of these two bands oscillates with the EDF length. When the intensities of two bands are nearly the same, the linewidth is at its local maximum, but at its local minimum when one of the bands dominates. The first minimum comes from the 1532-nm band intensity, which then grows more 


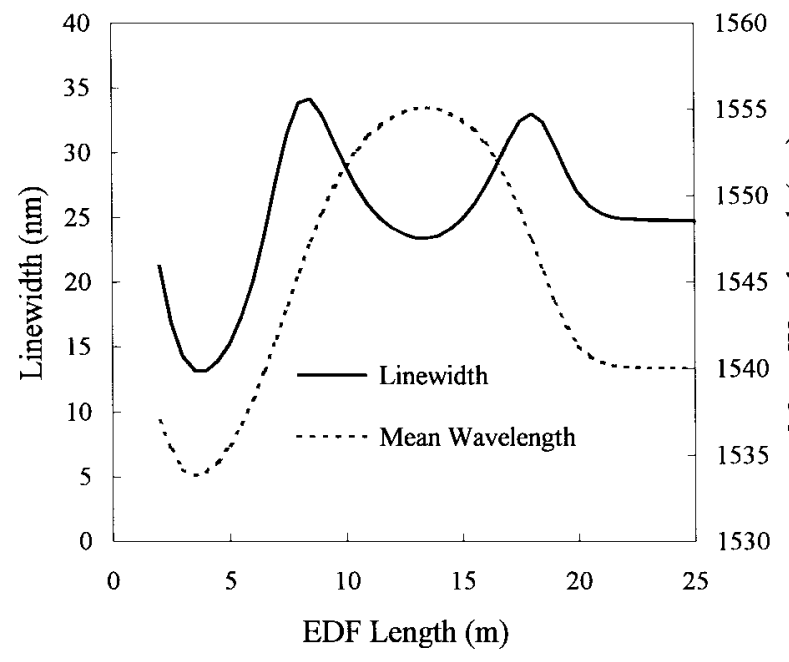

Fig. 6. Linewidth and mean wavelength variations with EDF length.

slowly than the 1558 -nm one until both balance; subsequently the 1558-nm band dominates and the second minimum results; then 1558-nm band decreases faster than 1532-nm one till both balance again. The final saturation level is the same as that of an SPB SFS.

\section{Effects of Pump Wavelength on Mean Wavelength}

Fig. 7(a) shows the effect of pump wavelength variation. The EDF length is chosen 12-m long as it is the optimal for $\partial \bar{\lambda}_{\text {source }} / \partial P_{\text {pump }} \approx 0$ at $80 \mathrm{~mW}$ pump. It is shown the minimum mean wavelength variation, i.e. $\partial \bar{\lambda}_{\text {source }} / \partial \lambda_{\text {pump }}=$ 0 , always occurs at the peak absorption wavelength, $\sim 976 \mathrm{~nm}$, regardless of pump power. Therefore, the second term in (1) that in fact constitutes the biggest contribution of mean wavelength stability as shown later can be eliminated. Unlike pump power dependence, described in Section III-B that the range of $\partial \bar{\lambda}_{\text {source }} / \partial P_{\text {pump }} \approx 0$ increases with pump power, there is no plateau region for $\partial \bar{\lambda}_{\text {source }} / \partial \lambda_{\text {pump }}$ dependence. As the pumping wavelength deviates from $976 \mathrm{~nm}$, the slope may become very large. A tradeoff between $\partial \bar{\lambda}_{\text {source }} / \partial P_{\text {pump }}$ and $\partial \bar{\lambda}_{\text {source }} / \partial \lambda_{\text {pump }}$ is therefore needed as implied in Fig. 7(a). For a commercial pumping diode, the uncertainty of lasing wavelength can be controlled to be within $\sim 0.1 \mathrm{~nm}$. Then, take the most commonly available wavelength $980 \mathrm{~nm}$ pumping as an example, the mean wavelength variation is expected to be $\sim 3 \mathrm{ppm}$ since the slope is $\sim 34 \mathrm{ppm} / \mathrm{nm}$. Fig. 7(b) shows the mean wavelength variation for various optimal EDF lengths pumped at their corresponding power. Note the mean wavelength variations are similar, and the DPB SFS still always has a $\partial \bar{\lambda}_{\text {source }} / \partial \lambda_{\text {pump }}=0$ operation at the wavelength of $976 \mathrm{~nm}$.

\section{E. Intrinsic Thermal Coefficients}

The effect of EDF length on intrinsic thermal coefficient pumped at $80 \mathrm{~mW}$ in various wavelengths is shown in Fig. 8 . Note that for each case there exist two EDF lengths, one long and the other is short, where the thermal coefficient is zero. At $980 \mathrm{~nm}$ pump, when the long EDF length $(\sim 12.5$ $\mathrm{m})$ is used, both $\partial \bar{\lambda}_{\text {source }} / \partial P_{\text {pump }} \approx 0(\sim 2.5 \mathrm{ppm} / \mathrm{mW})$ and

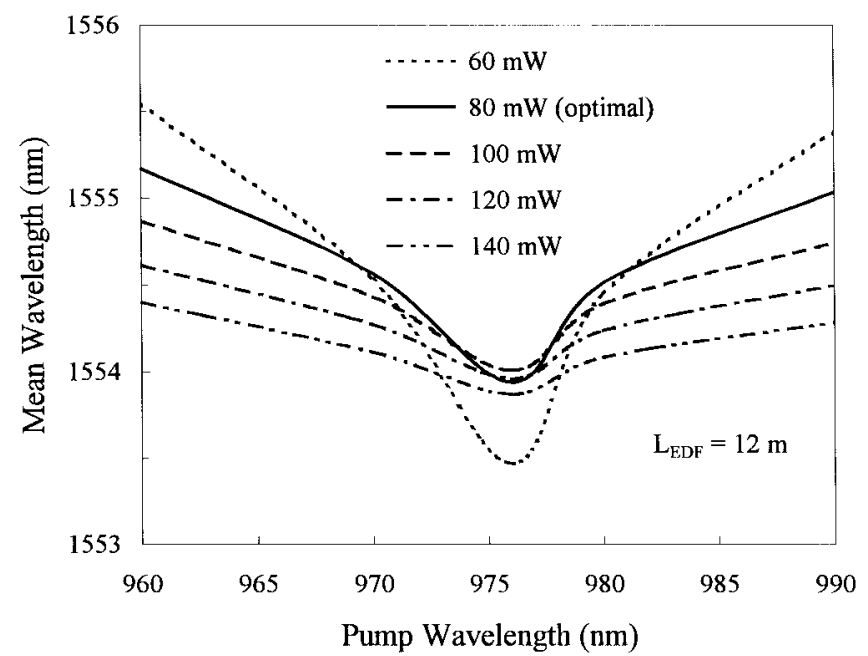

(a)

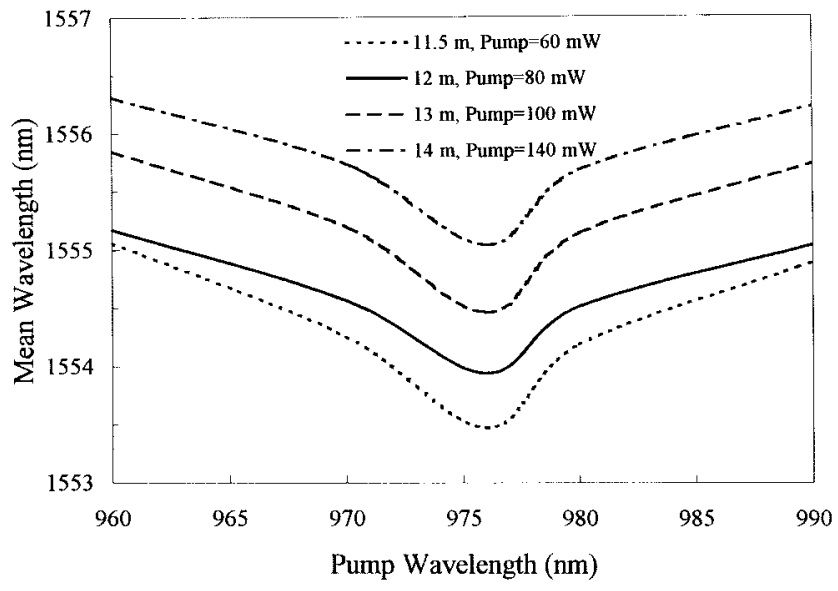

(b)

Fig. 7. Mean wavelength versus pump wavelength: (a) at various pump power and (b) for optimal EDF lengths and pump power.

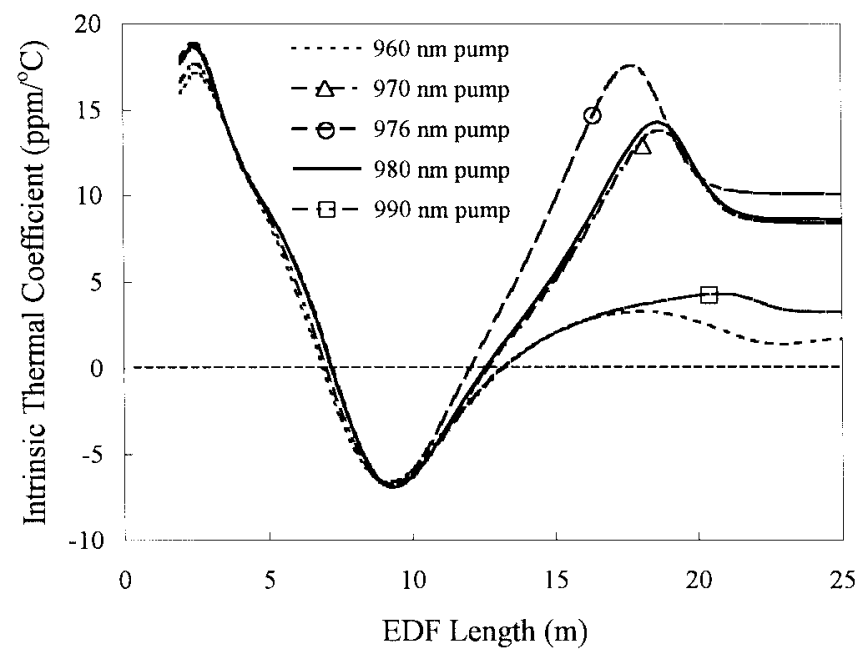

Fig. 8. Intrinsic thermal coefficient versus EDF lengths at various pump wavelengths.

$\partial \bar{\lambda}_{\text {source }} / \partial T_{\text {int. }}=0$ operations can be obtained. It is also shown that the intrinsic thermal coefficient reaches a saturated value when the EDF length is greater than some value for each pump wavelength. 


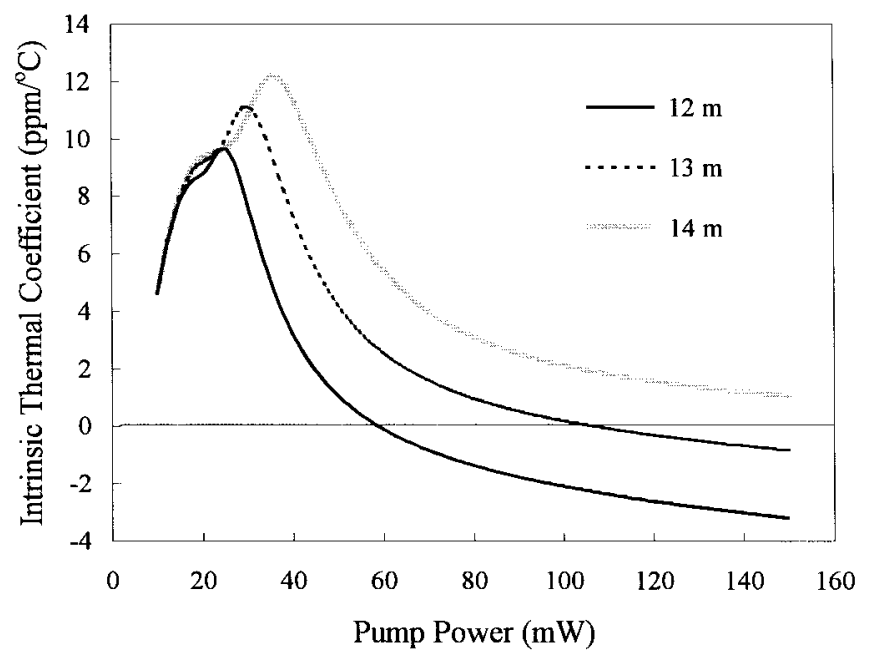

Fig. 9. Intrinsic thermal coefficient versus pump power at various optimal EDF lengths.

Fig. 9 shows the effect of pump power on intrinsic thermal coefficient. The EDF lengths are chosen as 12,13 and $14 \mathrm{~m}$ where $\partial \bar{\lambda}_{\text {source }} / \partial P_{\text {pump }} \approx 0$ occurs at the pump power of 80,100 and $140 \mathrm{~mW}$ respectively as shown in Fig. 2(b). It is seen that within a total power tolerance of $40 \mathrm{~mW}$ centered at their optimal pump power that has $\partial \bar{\lambda}_{\text {source }} / \partial P_{\text {pump }} \approx 0$, the absolute values of $\partial \bar{\lambda}_{\text {source }} / \partial T_{\text {int. are smaller than } 2}$ $\mathrm{ppm} /{ }^{\circ} \mathrm{C}$. Therefore, if the working temperature variation can be controlled within $0.1{ }^{\circ} \mathrm{C}$ accuracy, the mean wavelength variation due to intrinsic thermal effect can be below $\sim 0.2$ ppm.

\section{F. Minimizing the Overall}

The overall effects of fiber temperature, pump power, and pump wavelength at the optimal EDF length is estimated as follows. Let us take $80 \mathrm{~mW} 980 \mathrm{~nm}$ pump for a 12-m EDF as an example. The fiber temperature dependence is -1.4 $\mathrm{ppm} /{ }^{\circ} \mathrm{C}$, pump power dependence is $-2.4 \mathrm{ppm} / \mathrm{mW}$, and 34 $\mathrm{ppm} / \mathrm{nm}$ for pump wavelength dependence. The uncertainty is assumed $0.1{ }^{\circ} \mathrm{C}, 10 \mu \mathrm{W}$, and $0.1 \mathrm{~nm}$ for fiber temperature, pump power, and pump wavelength, respectively. Therefore, the overall wavelength stability is $\sim 3.4 \mathrm{ppm}$ by taking the square root of the summation of square of the three values. Note that the major contribution of the mean wavelength variation comes from the effect of the pump wavelength variation.

Since a $\partial \bar{\lambda}_{\text {source }} / \partial \lambda_{\text {pump }}=0$ operation can be obtained at the peak absorption wavelength $(976 \mathrm{~nm})$, the overall thermal effect can be minimized if one can minimize the pump power and intrinsic thermal effects. At reflectance of $90 \%$, it is found that if a 13-m-long EDF is pumped at $150 \mathrm{~mW}$, the fiber temperature dependence is only $-0.1 \mathrm{ppm} /{ }^{\circ} \mathrm{C}$, and the pump power dependence is only $-0.07 \mathrm{ppm} / \mathrm{mW}$. Therefore, under such condition, the DPB SFS can have a very stable operation. However, as the pump wavelength deviates from $976 \mathrm{~nm}$, although zero intrinsic thermal coefficient and pump power dependence can be obtained by properly choosing the pump power and EDF length, the overall thermal effect is essentially

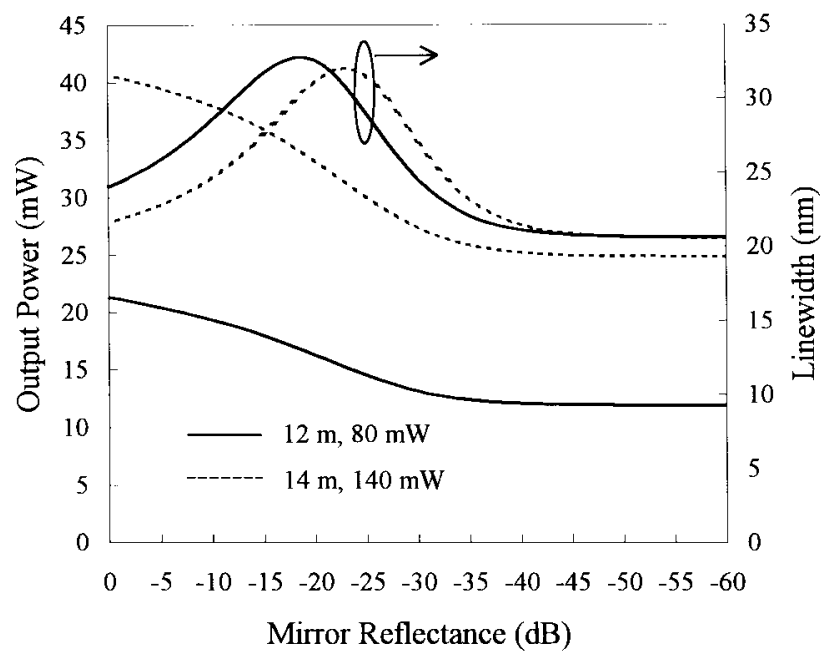

(a)

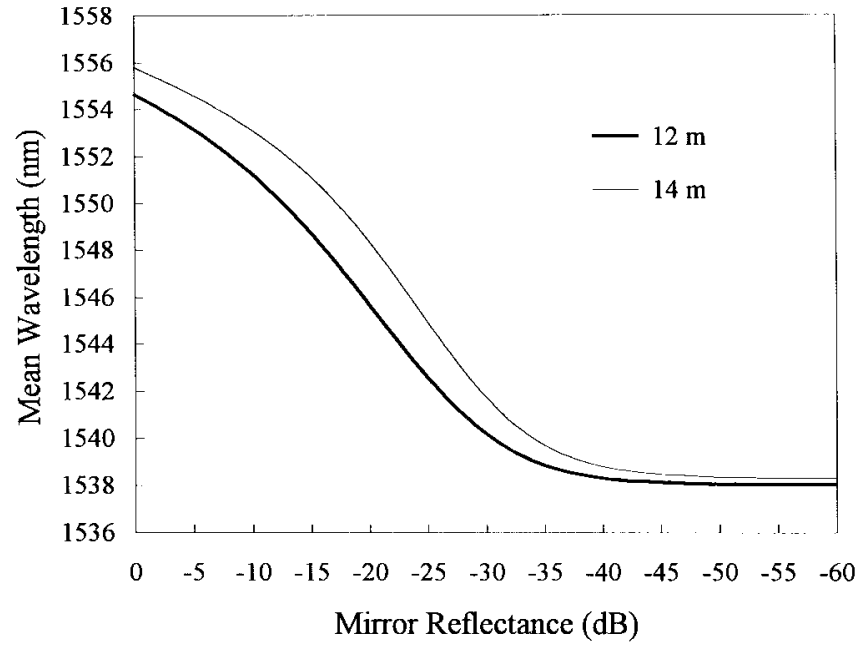

(b)

Fig. 10. Effects of mirror reflectance on (a) output power and linewidth and (b) mean wavelength.

determined by the pump wavelength effect. Therefore, the DPB SFS can have zero overall thermal coefficient only when pumped at its peak absorption wavelength.

\section{G. Effects of Mirror Reflectance}

The effects of mirror reflectance on output power, linewidth, and mean wavelength are shown in Fig. 10(a) and (b). It is seen as the reflectance becomes small $(<-40 \mathrm{~dB})$, both output power and linewidth become saturated since the DPB behaves like the SPB configuration. The output power increases with mirror reflectance. The linewidth is reduced in high and low reflectance, but has a maximum value in between. This linewidth variation can be understood from the evolution of spectra: as mirror reflectance increases from -60 to $0 \mathrm{~dB}$ the dominant mean wavelength gradually shifts from the 1532-nm band to the $1558-\mathrm{nm}$ band. It is interesting to note that, from Fig. 10(a), a reflectance of 3-4\% can also provide satisfactory high output power and a broad linewidth, suggesting that a cleaved fiber end may serve as mirror to achieve a stable 


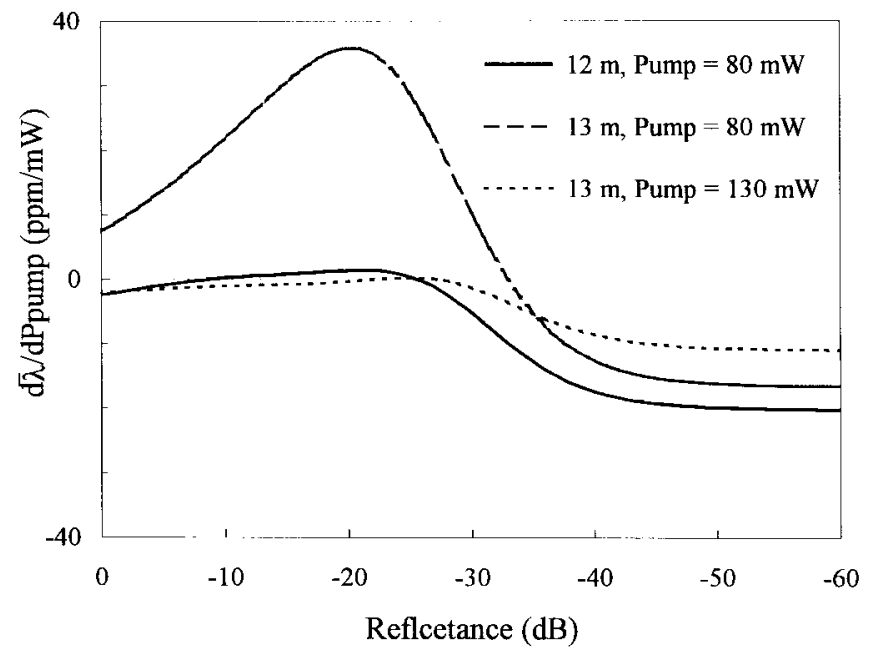

Fig. 11. Pump-power dependent mean wavelength stability versus mirror reflectance in several exemplary conditions.

DPB SFS. Simulation and experiment on this regard are being conducted.

Since mean wavelength stability is the most important characteristics for FOG applications, we have investigated to see if $\partial \bar{\lambda}_{\text {source }} / \partial P_{\text {pump }} \approx 0$ operation can also be obtained over some mirror reflectance range by adjusting pump power and EDF length. It is found that over some range of EDF length the shifting of mean wavelength of DPB and SPB SFS's are in opposite directions. By this and the fact that as reflectance becomes less the DPB SFS resembles more the SPB SFS, one can adjust the mirror reflectance to have $\partial \bar{\lambda}_{\text {source }} / \partial P_{\text {pump }}=0$ over that length range. A rule of thumb to have $\partial \bar{\lambda}_{\text {source }} / \partial P_{\text {pump }} \approx 0$ operation over a range of mirror reflectance is that $\partial \bar{\lambda}_{\text {source }} / \partial P_{\text {pump }}$ at $100 \%$ mirror reflectance should be slightly less than zero. For example, shown in Fig. 11 is the case for EDF lengths of 12 and $13 \mathrm{~m}$ at different pump power. The value of $\partial \bar{\lambda}_{\text {source }} / \partial P_{\text {pump }}$ first increases then decreases as the mirror reflectance decreases. At $12 \mathrm{~m}$ long EDF, $\partial \bar{\lambda}_{\text {source }} / \partial P_{\text {pump }}$ is less than $1 \mathrm{ppm} / \mathrm{mW}$ for reflectance between $\sim-10$ and $-25 \mathrm{~dB}$. At $13 \mathrm{~m}$ long EDF, it is seen that although $\partial \bar{\lambda}_{\text {source }} / \partial P_{\text {pump }} \approx 0$ exists at both pump power, only $130 \mathrm{~mW}$-pump shows $\partial \overline{\bar{\lambda}}_{\text {source }} / \partial P_{\text {pump }} \approx$ 0 exists over a large range of reflectance. In addition, the use of $12 \mathrm{~m}$ at $80 \mathrm{~mW}$ instead of $13 \mathrm{~m}$ at $80 \mathrm{~mW}$ is more practical due to its gradual variation with reflectance and double zero crossing.

\section{H. Effects of Feedback}

In all the above discussions, it is assumed that there is no feedback in the output fiber end. In practice, even when an isolator or an angled fiber end is used for feedback reduction, there still remains some residual feedback. It is therefore important to know to what extent the feedback can significantly affect the SFS's characteristics. Fig. 12(a) and (b) shows the variations of mean wavelength, linewidth and output power at different feedback levels. The shifting of mean wavelength can be explained from the evolution of ASE

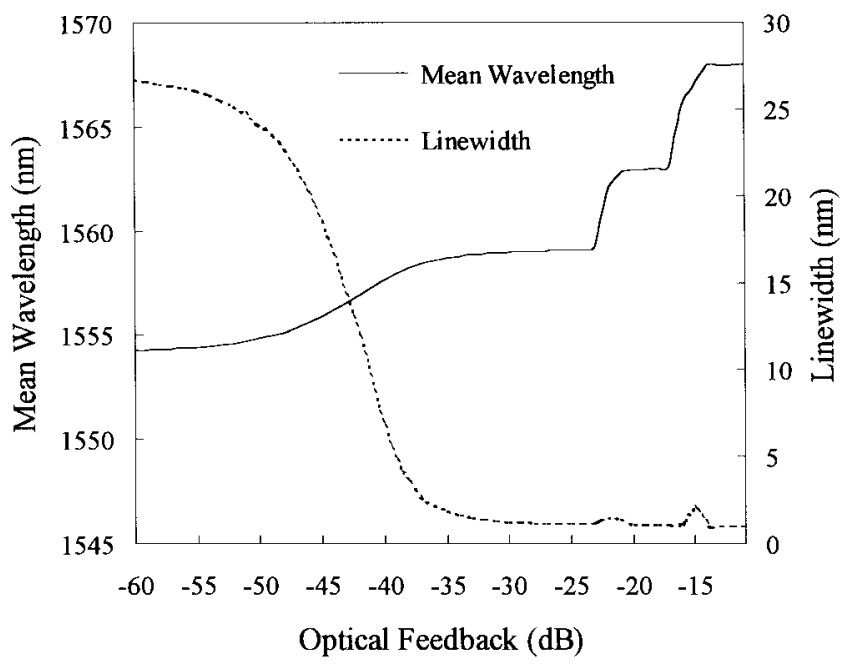

(a)

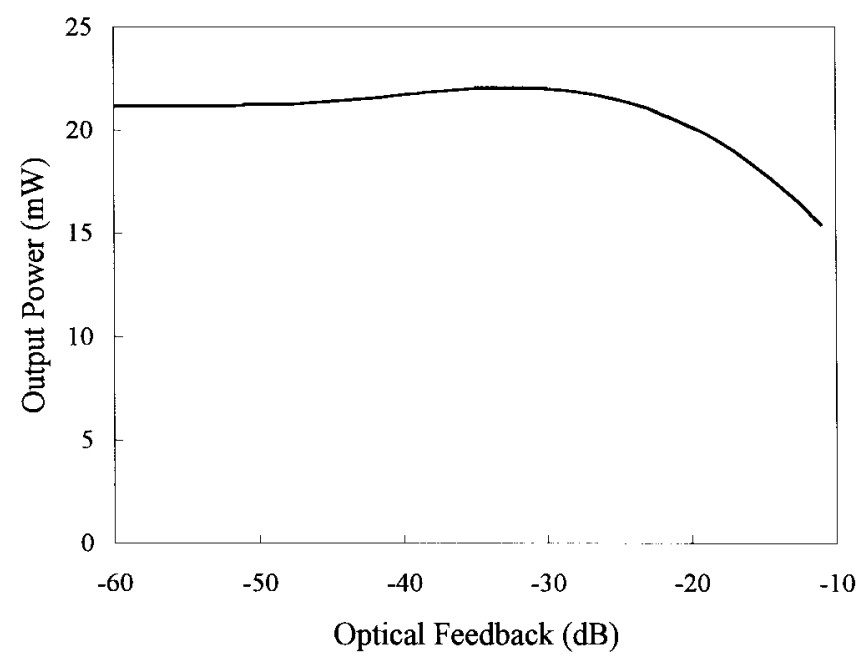

(b)

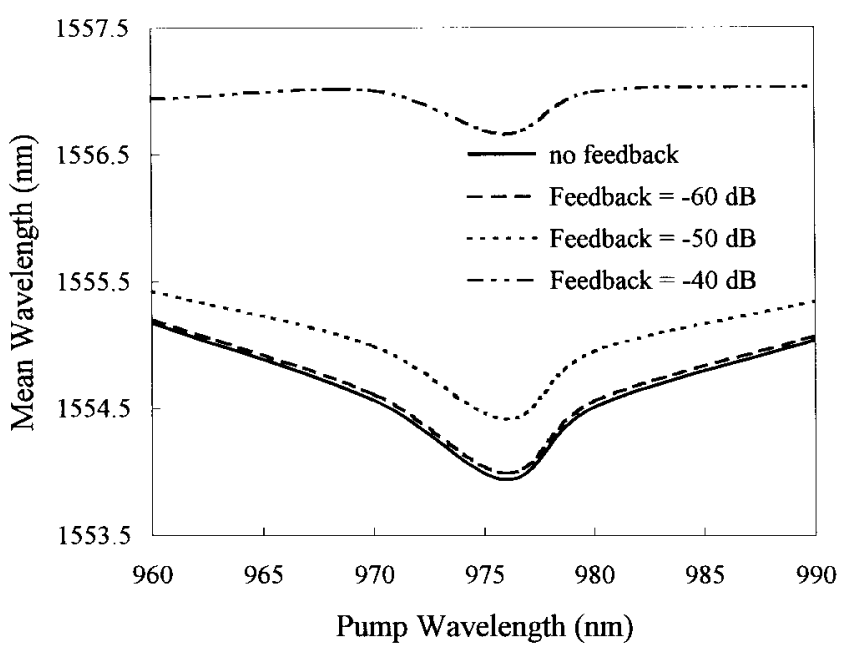

(c)

Fig. 12. Effects of optical feedback on (a) mean wavelength and linewidth, (b) output power, and (c) pump-wavelength dependent mean wavelength variation.

spectrum. For increasing optical feedback, the power ratio of $1558-\mathrm{nm}$ to $1532-\mathrm{nm}$ bands increases, thus causing the mean wavelength to shift toward long wavelength side. At the same time, a resonance cavity is forming that causes a 
marked decrease in linewidth. When the feedback is estimated larger than $-35 \mathrm{~dB}$, the lasing linewidth should have been much smaller than that shown in Fig. 12(a). This is because although the wavelength resolution employed in our simulation is suitable for modeling SFS's, it may not be applicable or even invalid as the feedback continues increasing to induce lasing. So is the reason for mean wavelength jump shown in the high feedback regimes. From these results, however, one finds that an optical feedback of less than $-60 \mathrm{~dB}$ is required to control the feedback induced mean wavelength stability to be less than $10 \mathrm{ppm} / \mathrm{dB}$. In Fig. 12(b), the output power first increases with feedback, and then decreases. The feedback ASE is reamplified in the EDF and becomes part of output signal when returned from the fiber mirror, therefore the output power increases with optical feedback. However, at an even higher feedback level, the feedback ASE also competes the same gain with the backward ASE, resulting a decrease of output power.

Fig. 12(c) shows the pump-wavelength dependent meanwavelength variation at different amount of optical feedback. The minimum mean wavelength occurs again at $976 \mathrm{~nm}$ pump. Note the mean wavelength increases with optical feedback for different pump wavelength. It is seen at $976 \mathrm{~nm}$ pump a shift up to $\sim 2.7 \mathrm{~nm}$ with optical feedback of $-40 \mathrm{~dB}$ as compared to that without feedback. The mean wavelength difference is negligibly small $(<0.1 \mathrm{~nm})$ between $-60 \mathrm{~dB}$ feedback and no feedback.

\section{Effects of $\mathrm{Er}^{3+}$ Concentration}

It has been shown that the concentration of ion pairs and clusters is a function of both erbium and codopant such as $\mathrm{Al}^{3+}$ concentrations [20]-[22]. As the $\mathrm{Er}^{3+}$ concentration increases or $\mathrm{Al}^{3+}$ content decreases, the fraction of ion pairs increases which deteriorates slope efficiency and threshold. Here we emphasize the effects of $\mathrm{Er}^{3+}$ concentration on the mean wavelength variation including $\partial \bar{\lambda}_{\text {source }} / \partial P_{\text {pump }}$, $\partial \bar{\lambda}_{\text {source }} / \partial \lambda_{\text {pump }}$, and $\partial \bar{\lambda}_{\text {source }} / \partial T_{\text {int. }}$. Three concentrations namely 80, 140 and 200 mole ppm are considered. Assuming a sizable $\mathrm{Al}^{3+}$ content, the corresponding fractions of unsaturable ion concentration used in the calculation are $1.7,3.0$ and $4.3 \%$, respectively. Without loss of generality, the other parameters are assumed to be the same as those given in Section III-A. Shown in Fig. 13(a) is the result of $\partial \bar{\lambda}_{\text {source }} / \partial P_{\text {pump }}$ dependence on the concentration at a fixed pump of $80 \mathrm{~mW}$. The results indicate that $\partial \bar{\lambda}_{\text {source }} / \partial P_{\text {pump }}=$ 0 operation can still be obtained by adjusting the EDF length, and the optimal EDF length decreases with erbium concentration.

Fig. 13(b) shows the intrinsic thermal coefficient versus the EDF lengths for different erbium concentration. The length of a zero intrinsic thermal coefficient decreases with the erbium concentration. Though there are two lengths for zero intrinsic thermal coefficient, one needs to examine these figures to determine which length is more suitable for minimizing the total results of pump power and temperature effects.

Fig. 13(c) shows the pump-wavelength dependent mean wavelength variation. The EDF lengths with zero pump power dependence at $80 \mathrm{~mW}$ pump shown in Fig. 13(a) are chosen

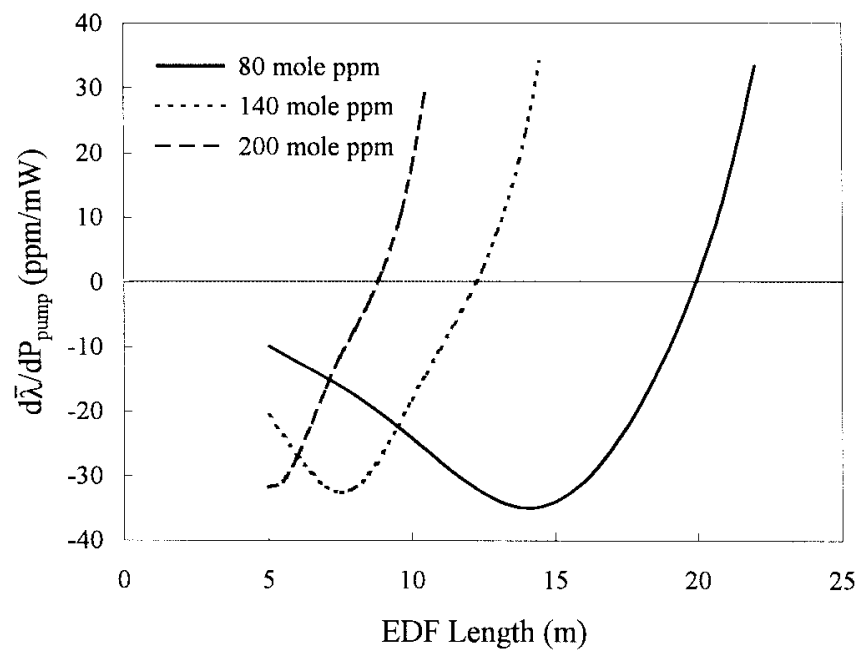

(a)

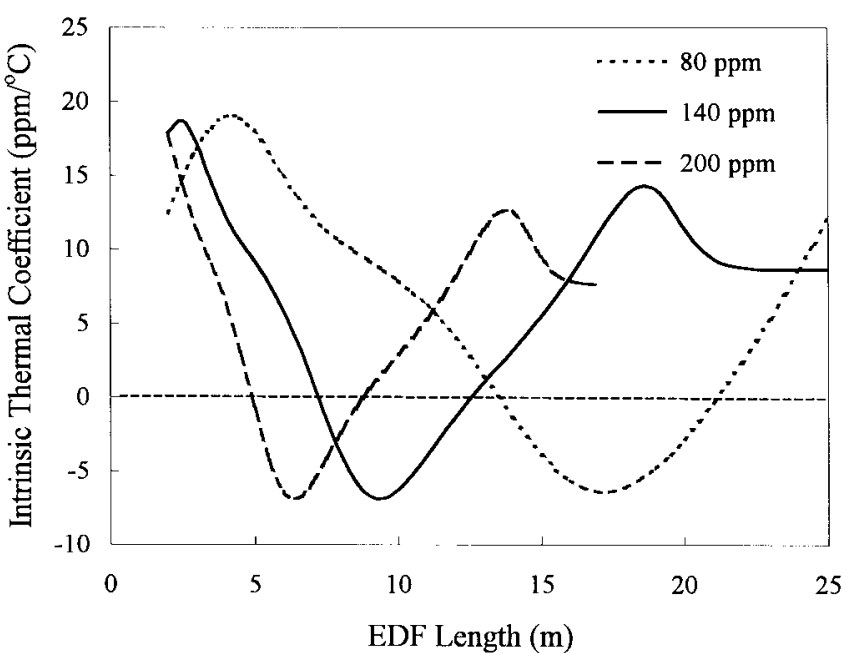

(b)

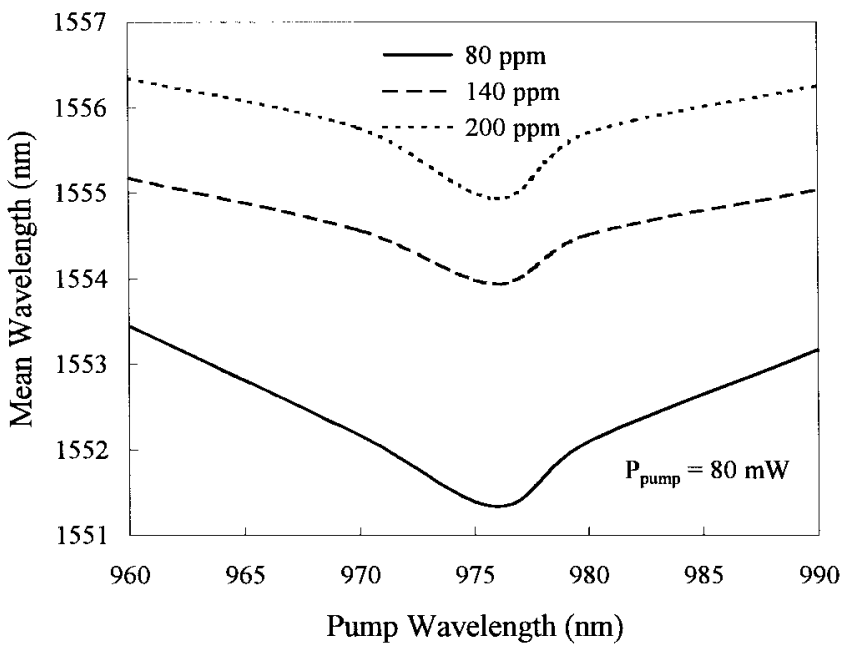

(c)

Fig. 13. Effects of erbium concentration on (a) pump-power dependent mean wavelength variation, (b) intrinsic thermal coefficient, and (c) pump-wavelength dependent mean wavelength variation based on optimal conditions of (a).

for these three erbium concentrations. It is shown that the minimum mean wavelength also occurs at pump wavelength of $976 \mathrm{~nm}$ for different erbium concentrations. 


\section{CONCLUSION}

We have analyzed the characteristics of an Er-doped SFS pumped near $980 \mathrm{~nm}$ in DPB configuration in details. The results can be summarized as the following. A $\partial \bar{\lambda}_{\text {source }} / \partial P_{\text {pump }}=0$ or $\partial \bar{\lambda}_{\text {source }} / \partial P_{\text {pump }} \approx 0$ operation can be obtained in high-pump regions by properly adjusting EDF length and pump power. Such an operation exists either over a wide pump power range for a suitable EDF length [cf., Fig. 2(a)] or over a large mirror reflectance range for a suitable pump (cf., Fig. 11). In addition, the tolerance of optimal EDF length can be increased by properly increasing pump power and EDF length [cf., Fig. 2(b)]. Very low intrinsic thermal coefficients can be obtained with proper pump power and EDF lengths (cf., Fig. 9) and over various pump wavelengths (cf., Fig. 8). As for pump wavelength dependence, which is the dominant factor, the minimum mean wavelength variation always occurs at the peak absorption wavelength of $\sim 976$ nm, regardless of various optimal conditions [cf., Fig. 7(b)], feedbacks [cf.,, Fig. 12(c)] and erbium concentrations [cf., Fig. 13(c)]. Therefore, the overall thermal effect can be minimized by first setting the pumping wavelength at $\sim 976$ $\mathrm{nm}$, and then finding an appropriate condition that leads to both minimum pump-power dependence and minimum intrinsic thermal coefficient. A very stable DPB SFS is numerically shown feasible.

An SFS in DPB configuration is also shown capable of providing high output power, good pump efficiencies and more than enough linewidth over a large range of EDF length at various mirror reflectance, indicating the feasibility of serving three-axis FOG's. The study of the effect of optical feedback on mean wavelength stability shows that the characteristics remain nearly unchanged as an isolator with isolation greater than $60 \mathrm{~dB}$ is employed. From these results, it is expected that an SFS in DPB configuration is potentially a desirable light source for the navigation-grade FOG applications.

\section{REFERENCES}

[1] H. C. Lefevre, The Fiber-Optic Gyroscope. Norwood, MA: Artech House, 1993

[2] P. R. Morkel, R. I. Laming, and D. N. Payne, "Noise characteristics of high-power doped-fiber superluminescent sources," Electron. Lett., vol. 26, pp. 96-98, 1990

[3] P. F. Wysocki, M. J. F. Digonnet, B. Y. Kim, and H. J. Shaw, "Characteristics of Erbium-doped superfluorescent fiber sources for interferometric sensor applications," J. Lightwave Technol., vol. 12, pp. 550-567, 1994

[4] D. C. Hall, W. K. Burns, and R. P. Moeller, "High-stability Er-doped superfluorescent fiber sources," J. Lightwave Technol., vol. 13, pp. $1452-1460,1995$.

[5] P. F. Wysocki, M. J. F. Digonnet, and B. Y. Kim, "Wavelength stability of a high-output, broadband, Er-doped superfluorescent fiber source pumped near 980 nm," Opt. Lett., vol. 16, pp. 961-963, 1991.
[6] D. C. Hall and W. K. Burns, "Wavelength stability optimization in Er-doped superfluorescent fiber sources," Electron. Lett., vol. 30, pp. 653-654, 1994.

[7] L. A. Wang, and C. D. Chen, "Stable and broadband Er-doped superfluorescent fiber sources utilizing double-pass backward configuration," Electron. Lett., vol. 32, pp. 1815-1817, 1996.

[8] K. Iwatsuki, "Er-doped superfluorescent fiber laser pumped by $1.48 \mu \mathrm{m}$ laser diode," IEEE Photon. Technol. Lett., vol. 2, pp. 237-238, 1990.

[9] P. F. Wysocki, K. Fesler, K. Liu, M. J. F. Digonnet, and B. Y. Kim, "Spectrum thermal stability of Nd- and Er-doped fiber sources," in Proc. Soc. Photo-Opt. Instrum. Eng., 1990, vol. 1373, pp. 234-245.

[10] K. Liu, M. Digonnet, H. J. Shaw, B. J. Ainslie, and S. P. Craig, "10 $\mathrm{mW}$ superfluorescent single-mode fiber source at $1060 \mathrm{~nm}$," Electron. Lett., vol. 23, pp. 1320-1321, 1987.

[11] P. F. Wysocki, M. J. F. Digonnet, and B. Y. Kim, "Spectral characteristics of high-power $1.5 \mu \mathrm{m}$ broad-band superluminescent fiber sources," IEEE Photon. Technol. Lett., vol. 2, pp. 178-180, 1990.

[12] I. N. Duling III, W. K. Burns, and L. Goldberg, "High-power superfluorescent fiber source," Opt. Lett., vol. 15, pp. 33-35, 1990.

[13] I. N. Duling III, R. P. Moeller, W. K. Burns, C. A. Villarruel, L. Goldberg, E. Snitzer, and H. Po, "Output characteristics of diode pumped fiber ASE sources," IEEE J. Quantum Electron., vol. 27, pp. 995-1003, 1991.

[14] L. A. Wang and C. D. Chen, "Characteristics comparison of Er-doped double pass superfluorescent fiber sources pumped near $980 \mathrm{~nm}$," IEEE Photon. Technol. Lett., vol. 9, pp. 446-448, 1997.

[15] "Comparison of efficiency and output power of optimal Er-doped superfluorescent fiber sources in different configurations," Electron. Lett., vol. 33, pp. 703-704, 1997.

[16] R. Paschotta, J. Nilsson, A. C. Tropper, and D. C. Hanna, "Efficient superfluorescent ligh source with broad bandwidth," IEEE J. Select. Topics Quantum Electron., vol. 3, pp. 1097-1099, 1997.

[17] S. D. Dyer and K. B. Rochford, "Spectral tailoring of erbium superfluorescent fiber source," Electron. Lett., vol. 34, pp. 1137-1139, 1998.

[18] P. F. Wysocki, R. F. Kalman, M. J. F. Digonnet, and B. Y. Kim "A comparison of $1.48 \mu \mathrm{m}$ and $980 \mathrm{~nm}$ pumping for Er-doped superfluorescent fiber sources," in Proc. Soc. Photo-Opt. Instrum. Eng., 1991, vol. 1581, pp. 40-57.

[19] P. F. Wysocki, J. L. Wagener, M. J. F. Digonnet and H. J. Shaw, "Evidence and modeling of paired ions and other loss mechanisms in erbium-doped silica fibers," Proc. Soc. Photo-Opt. Instrum. Eng., vol. 1789, pp. 66-79, 1992.

[20] E. Delevaque, T. Georges, M. Monerie, P. Lamouler, and J.-F. Bayon, "Modeling of pair-induced quenching in erbium-doped silicate fibers," IEEE Photon. Technol. Lett., vol. 5, pp. 73-75, 1993.

[21] J. L. Wagener, P. F. Wysocki, M. J. F. Digonnet, and H. J. Shaw, "Effects of concentration and clusters in erbium-doped fiber lasers," Opt. Lett. vol. 18, pp. 2014-2016, 1993.

[22] R. S. Quimby, W. J. Miniscalco, and B. Thompson, "Upconversion and 980-nm excited-state absorption in erbium-doped glass," in Proc. Soc. Photo-Opt. Instrum. Eng., 1992, vol. 1789, pp. 50-57.

Lon A. Wang, photograph and biography not available at the time of publication.

C. D. Su, photograph and biography not available at the time of publication. 